\title{
QUELLE DEMANDE DE FORMALISATION LÉGALE DES DROITS FONCIERS ?
}

Éclairage à partir d'une commune des Hautes Terres malgaches

Céline Boué, Pierre-Marie Bosc, Jean-Philippe Colin

Armand Colin | « Revue Tiers Monde »

2016/3 N²26-227 | pages 37 à 64

ISSN 1293-8882

ISBN 9782200930752

Article disponible en ligne à l'adresse :

http://www.cairn.info/revue-tiers-monde-2016-3-page-37.htm

\section{Pour citer cet article :}

Céline Boué et al., "Quelle demande de formalisation légale des droits fonciers ? Éclairage à partir d'une commune des Hautes Terres malgaches », Revue Tiers Monde 2016/3 ( ${ }^{\circ}$ 226-227), p. 37-64.

Distribution électronique Cairn.info pour Armand Colin.

(C) Armand Colin. Tous droits réservés pour tous pays.

La reproduction ou représentation de cet article, notamment par photocopie, n'est autorisée que dans les limites des conditions générales d'utilisation du site ou, le cas échéant, des conditions générales de la licence souscrite par votre établissement. Toute autre reproduction ou représentation, en tout ou partie, sous quelque forme et de quelque manière que ce soit, est interdite sauf accord préalable et écrit de l'éditeur, en dehors des cas prévus par la législation en vigueur en France. Il est précisé que son stockage dans une base de données est également interdit. 


\section{Quelle demande de formalisation légale des droits fonciers?}

\section{Éclairage à partir d'une commune des Hautes Terres malgaches}

Céline Boué

Pierre-Marie Bosc

Jean-Philippe Colin

\section{MOTS-CLÉS}

Droits de propriété, formalisation des droits, certification foncière, sécurisation foncière, Madagascar.

\section{RÉSUMÉ}

Ce texte explore les déterminants de l'engagement, ou non, des ménages agricoles dans le processus de certification issu de la réforme foncière malgache de 2005. Nos travaux, conduits dans une commune rurale des Hautes Terres, montrent (i) que la principale motivation à la demande de certificat est la recherche d'une sécurisation des droits fonciers en soi, indépendamment d'éventuelles perspectives d'investissements futurs ou de mise sur le marché de la terre, (ii) que les caractéristiques de la parcelle influent tant sur la décision de certifier que sur le choix des parcelles à certifier 


\section{Introduction}

$\mathrm{D}$ ès le $\mathrm{XIX}^{\mathrm{e}}$ siècle, les puissances coloniales ont mis en place des procédures de formalisation des droits dans leurs territoires ultramarins (Acte Torrens en Australie en 1858, Sénatus-Consulte en Algérie en 1863, Plan Swynnerton au Kenya en 1954, notamment), une politique que les gouvernements des États devenus indépendants ont ensuite poursuivie. Transformer les droits « coutumiers » en droits privés individuels, reconnus légalement et enregistrés par les services de l'État, induirait une sécurisation foncière propice aux investissements, ainsi que l'activation des marchés fonciers, indispensable pour assurer une allocation optimale de la terre (Colin et al., 2009). Le constat fréquemment tiré des limites des politiques de formalisation des droits par l'émission de titres de propriété privée (faisabilité financière, technique et politique, biais de sélection en faveur de certains acteurs) ont conduit certains experts à recommander un assouplissement des procédures de reconnaissance des droits, à travers l'émission de certificats fonciers et non plus de titres fonciers (Van den Brink et al., 2006 ; Deininger \& Feder, 2009). La reconnaissance légale des droits fonciers gagnerait en efficacité et en équité si elle était placée sous la responsabilité des collectivités locales, en impliquant les populations. La réforme foncière malgache de 2005 illustre ce nouveau référentiel. L'État malgache indépendant (1960) avait repris à son compte le principe de domanialité. Le passage du domaine de l'État à un statut privé n'était possible qu'à l'issue d'une procédure d'immatriculation, sous la responsabilité des services déconcentrés de l'État - les services fonciers implantés au niveau de chaque région. Cette procédure n'a touché qu’une faible proportion du territoire national et est reconnue comme déficiente du fait de son coût, des pratiques de corruption, de la non actualisation des transferts fonciers et des mauvaises conditions de conservation des livres fonciers (PNF, 2007 ; Teyssier et al., 2007). Dans les années 2000, un diagnostic de crise foncière et domaniale fait consensus au sein des institutions de développement et d'une partie de l'administration malgache ${ }^{1}$ et,

1 La majeure partie de l'administration des Domaines est cependant restée réticente au changement. L'élaboration de la nouvelle Lettre de politique foncière (LPF) 2015-2030 a récemment donné lieu à des mouvements de grève de syndicats des agents de cette administration, qui ont conduit à une remise en cause radicale de la nouvelle LPF. La société civile dénonce alors la marginalisation des communes 
en 2005, est votée la loi 2005-019, qui opère une rupture dans la trajectoire institutionnelle. Elle met fin au principe de domanialité, en reconnaissant légalement la « propriété privée non titrée » (PPNT), formalisable par un certificat foncier. L'émission et la gestion des certificats fonciers sont déléguées à des «guichets fonciers » décentralisés et placés sous l'autorité des communes, au terme d'une procédure contradictoire de validation locale (PNF, 2007 ; Teyssier et al., 2009). La procédure d'immatriculation reste cependant en vigueur, l'émission du titre restant la prérogative des services des Domaines.

Dans la littérature économique, la question de la formalisation des droits fonciers est principalement abordée à travers l'impact des politiques de titrage sur les investissements productifs, qui seraient stimulés par la sécurité apportée par le titre (Feder \& Onchan, 1987 ; Besley, 1995 ; Feder \& Nishio, 1999 ; Smith, 2004). L'objet central de cet article porte, en amont, sur la demande de sécurisation des acteurs à travers la formalisation de leurs droits de propriété - souvent considérée, dans la littérature, comme allant de soi.

Notre analyse s'appuie sur des investigations conduites dans la commune rurale de Faratsiho qui abrite l'un des premiers guichets fonciers mis en place (2006), financé par le Millenium Challenge Account (MCA). En septembre 2013, 2159 demandes de certificats avaient été reçues, et 1937 certificats délivrés. Les données de première main ont été collectées lors d'un travail empirique (Boué, 2013), qui a été mené sur un total de 15 mois, de mai 2008 à novembre 2011. Au-delà des apports propres à une telle immersion, notre analyse s'appuie sur deux types de données plus systématiques : (i) des entretiens approfondis ${ }^{2}$ conduits auprès de 85 ménages $^{3}$, portant sur les pratiques foncières, les sources d'insécurité, les dispositifs de reconnaissance des droits

avec un recul dans le processus de décentralisation ainsi que le non respect du principe de la transparence dans l'élaboration de la LPF (http://lobservateur-mada. mg/resolution-de-la-greve-des-employes-des-domaines-le-s-i-f-denonce-la-rectification-hative-de-la-lettre-de-politique-fonciere/).

2 Dans la suite du texte, nous utiliserons le terme " entretiens " lorsque nous nous réfèrerons à ces données.

3 Les ménages merina sont monogames. Les terroirs rizicoles situés dans les Hautes Terres tendent depuis plusieurs décennies à être appropriés par un individu ou une famille restreinte et sont ainsi de moins en moins soumis à une gestion collective sous I'autorité des pouvoirs lignagers (Ottino, 1998 ; Droy et al., 2010). 
(entendus dans l'acception usuelle en sciences sociales au sens d'actions socialement autorisées sur un bien) ${ }^{4}$; (ii) une enquête par questionnaire auprès de 405 ménages $^{5}$ ( 2497 parcelles au total), qui surreprésentait volontairement les ménages possédant au moins un certificat ${ }^{6}$ (2O3, contre 202 n'en ayant aucun). En moyenne, les ménages entrés dans la procédure disposaient de 2,5 certificats (de 1 à 15 ) et avaient certifié, au moment de l'enquête, $50 \%$ des parcelles qu'ils possédaient (de $10 \%$ à $100 \%$ ).

La commune de Faratsiho, située dans les Hautes Terres centrales, à $87 \mathrm{~km}$ au nord-est d'Anstirabe (fig. 1), comprend dix-huit villages (fokontany). La région se caractérise par de très faibles mouvements migratoires, par diverses pratiques de formalisation mobilisant les aînés et les autorités administratives locales et par des droits individualisés, avec cependant une gestion plus « collective » en intrafamilial. Contrairement à d'autres zones de Madagascar, la commune étudiée n’est pas concernée par les investissements fonciers à grande échelle. Tous les enquêtés sont merina ${ }^{7}$ et, à de rares exceptions près, riziculteurs. La rizière est le capital productif le plus important dans cette agriculture familiale, essentiellement tournée vers l'autoconsommation (en moyenne 24,3 ares de rizières par ménage dans notre échantillon). Les familles, structurées autour de ménages monogames qui peuvent cohabiter avec la génération précédente, exploitent en outre les terres de colline (tanety), destinées principalement au haricot, au maïs, au soja et aux tubercules.

4 Plutôt qu'aux " droits ", nous ferons référence aux " faisceaux de droits " dont les "fibres" sont susceptibles d'être contrôlées par différents individus : droits d'usage, de déléguer l'usage de la terre à titre marchand ou non marchand, d'aliéner à travers des transferts définitifs, de réguler le droits des autres, etc. (Alchian \& Demsetz, 1973 ; Schlager \& Ostrom, 1992 ; Colin, 2008).

5 Nous utiliserons le terme " enquêtes par questionnaire " pour faire référence à ces données.

6 En février 2010, 994 certificats avaient été délivrés au niveau des 7 fokontany enquêtés, soit un taux de couverture de la certification d'environ $18 \%$. Nous pondérons donc nos résultats (en le précisant dans le texte) lorsque nous les inférons au niveau de la commune.

7 Ethnie dominante des Hautes Terres, détenant historiquement les pouvoirs politique et administratif de l'île. 


\section{Figure 1. Localisation de la région d'étude}

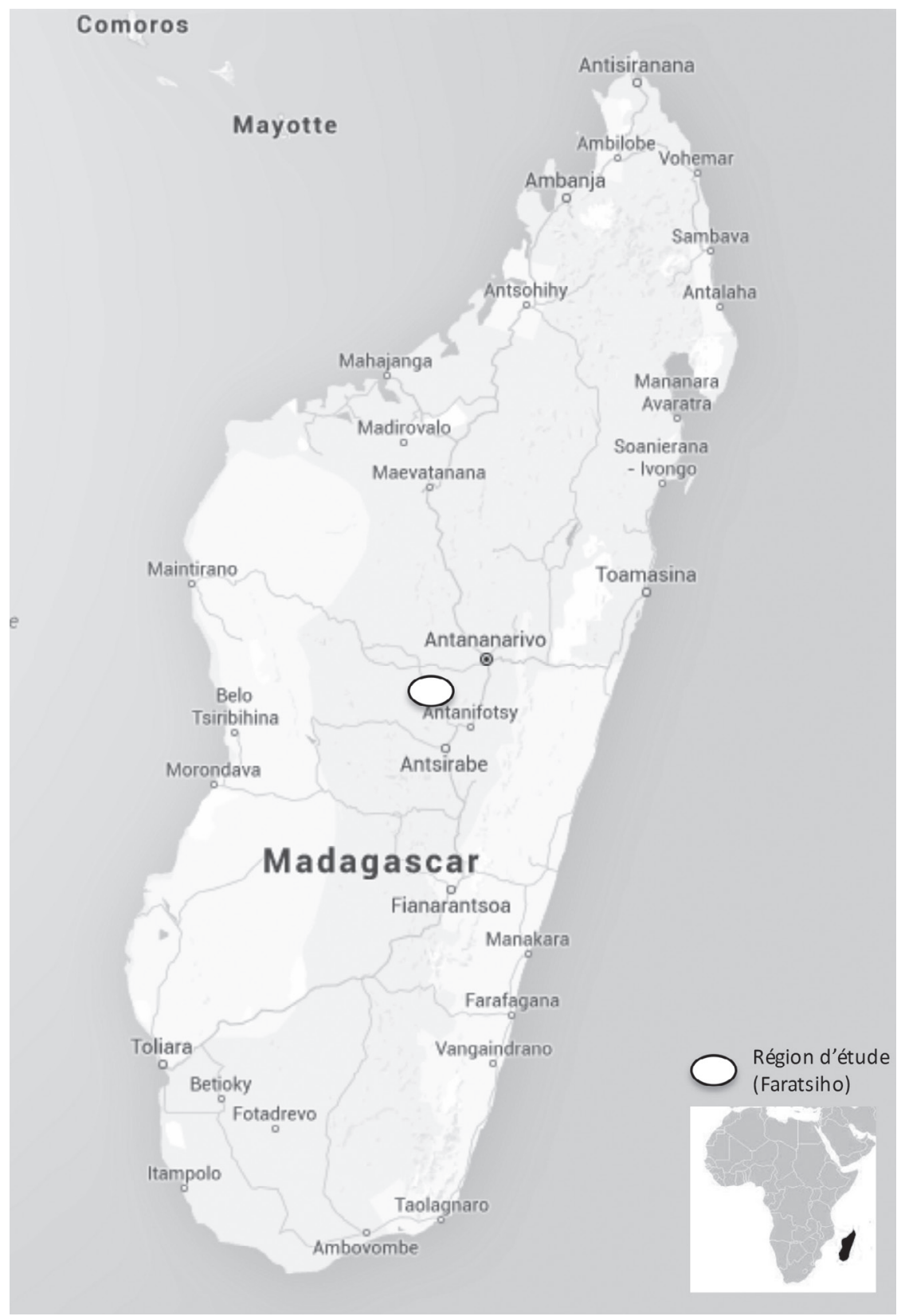

Source : Auteurs, 2016. 
La première section de ce texte explore les motivations du recours à la certification. La seconde section précise les déterminants du choix des parcelles à certifier. La dernière section propose une discussion de la relativement faible mobilisation de la procédure de certification quatre années après l'installation du guichet foncier et met l'accent sur l'importance du niveau parcelle pour l'analyse des déterminants de la certification.

\section{Les déterminants de la demande de certificats fonciers}

Dès nos premiers entretiens, la recherche d'une sécurisation du patrimoine familial est apparue comme la principale motivation de la demande de certification (tab. 1).

\section{Tableau 1. Avantages associés à la détention d'un certificat (55 enquêtés, 2008)}

\begin{tabular}{l|c} 
Principal avantage du certificat selon les enquêtés & Nombre de ménages \\
\hline Protège d'éventuelles contestations sur la parcelle & $31(56 \%)$ \\
\hline Facilite l'héritage & $16(29 \%)$ \\
\hline Possède une valeur légale en cas de conflit & $8(15 \%)$ \\
\hline Peut être utilisé comme garantie pour des crédits & o \\
\hline Motive un investissement futur sur cette parcelle & O \\
\hline Facilite la vente ou la location de la parcelle & O \\
\hline Permet de vendre plus cher les parcelles certifiées & o \\
\hline Total & $55(100 \%)$ \\
\hline
\end{tabular}

Source : Données issues d'entretiens préliminaires (Boué, 2008).

L'insécurité foncière relève d'une perception beaucoup plus que des faits. Près d'un enquêté sur deux a fait état de litiges dont il avait eu connaissance, mais 16 parcelles seulement sur les 2497 de l'enquête par questionnaire (o,6 \%) avaient fait l'objet de tensions ou de conflits.

Dans cette section, nous discutons des attentes économiques comme justification de la certification. En premier lieu, le recours à la certification ne peut pas être interprété comme visant à faciliter un accès au crédit formel. Seule l'une des deux institutions financières présentes à Faratsiho (une 
institution de microfinance : l'Ombana Tahiry Ifampisambonara Vola - Otiv) accepte le certificat en garantie ${ }^{8}$. Selon son directeur, en janvier 2010, sur 290 crédits octroyés depuis l'ouverture de l'institution en novembre 2006, 3o seulement avaient fait l'objet d'un dépôt de certificat en garantie. Parmi les 405 ménages enquêtés, une seule demande de certificat avait été motivée par une perspective d'accès au crédit formel. De façon générale, le marché du crédit formel est très faiblement développé dans la commune ${ }^{9}$, seuls les ménages les plus aisés et ayant des revenus d'activités extra-agricoles y ont accès. Par ailleurs, la détention de garanties foncières légales n’est pas déterminante dans l'accès au crédit, l'Otiv acceptant d'autres types de justificatifs de propriété (taratasy $\left.{ }^{10}\right)$ (Zombre, 2013).

En second lieu, aucun enquêté ne justifie la demande de certificat par le projet de vendre ultérieurement la terre. Les droits de propriété du vendeur sont rarement légalement enregistrés. Sur 907 parcelles achetées, seules 37 (4\%) étaient documentées avant le transfert par un titre foncier (35) ou un certificat foncier (2). Aucun ménage n'évoque le fait que la parcelle soit titrée ou certifiée comme déterminant du choix pour la mettre en vente. De nombreuses études empiriques menées dans d'autres contextes ont montré l'absence de relation systématique entre formalisation légale et développement des marchés fonciers (Atwood, 1990 ; Platteau, 1996 ; Place \& Migot-Adholla, 1998) et le développement de marchés fonciers dynamiques sans formalisation légale des droits (Platteau, 1996 ; Colin \& Ayouz, 2006 ; pour Madagascar, Burnod et al., 2014). Dans notre étude, le marché de l'achatvente apparaît également comme actif malgré le faible nombre de parcelles formalisées légalement. Les acheteurs n’imposent pas de sécurisation légale préalable, les coûts de recherche d'information quant aux droits de propriété des cédants étant faibles compte tenu de l'importance des relations de connaissances interpersonnelles et du marché fortement développé dans le cadre familial (sur les caractéristiques du marché foncier dans la localité : Boué \& Colin, 2015). Des liens entre la demande de certificat et le marché de

8 En 2015, la majorité des agences de financement est encore réticente quant à I'usage du certificat foncier comme garantie (http://www.observatoire-foncier.mg/ article-108/\#sthash.YtmxGU24.dpufhttp://www.observato/).

9 Les travaux de l'Observatoire du foncier montrent également que seuls $5 \%$ des 1800 ménages avaient eu recours au crédit en 2011 (Burnod et al., 2014).

10 La certification et l'immatriculation sont qualifiées dans ce texte de formalisation légale, les autres élaborations de documents étant qualifiées de formalisation locale. 
l'achat-vente existent bien, mais portent sur une recherche de sécurisation des transactions passées : le certificat permet de se protéger d'éventuels risques de remise en cause de la transaction (cf. infra).

En troisième lieu, la sécurisation des droits sur la parcelle en vue de la céder en faire-valoir indirect (FVI) n’est jamais évoquée par les enquêtés comme motivation de la demande de certificat. Les cessions en location renvoient à un besoin financier urgent, le paiement de la rente s'effectuant avant la mise en culture. Dans ces conditions, entrer dans une démarche de certification coûteuse et longue n'a que peu de sens. Les cessions en métayage interviennent au sein d'une fratrie afin d'éviter qu'une parcelle héritée reste en friche. Dans la majorité des contrats de FVI, la relation est basée sur la confiance. Dans le cas de tanety éloignées, la cession en FVI peut même être perçue comme un dispositif de sécurisation, en prévenant d'éventuels empiètements de la part des voisins. Si les cédants envisagent une contestation de leurs droits de propriété par le futur tenancier, d'autres dispositifs de sécurisation que le certificat sont mobilisés : sélection du partenaire, limitation de la durée de la cession, formalisation du contrat par acte sous-seing privé.

En quatrième lieu, le certificat n'est pas demandé dans la perspective d'un futur investissement - verger, aménagement pour l'irrigation ou le drainage (Barrows \& Roth, 1990 ; Migot-Adholla et al., 1991 ; Jacoby \& Minten, 2007). Par contre, il arrive qu'un certificat soit demandé pour protéger un investissement passé (Burnod et al., 2014) ; le plus souvent une tanety pour laquelle un système d'irrigation a été mis en place. La causalité sécurisation/investissement est en fait parfois inversée : dans certains contextes, l'investissement sur la terre contribue à établir, renforcer ou sécuriser le droit sur la terre (Besley, 1995 ; Sjaastad \& Bromley, 1997 ; Colin et al., 2009). On retrouve cette logique dans notre étude, l'investissement régulier en travail et la reconnaissance sociale qui lui est associée générant une certaine sécurité foncière sur une parcelle donnée, ce qui contribue à expliquer un manque d'intérêt pour une formalisation légale des droits - nous y reviendrons. 


\section{Les déterminants du choix des parcelles à certifier}

Peu d'enquêtés entrés dans le programme de certification avaient certifié l'ensemble des parcelles possédées. La question de la demande ou non de certification foncière se pose donc non seulement au niveau des ménages, mais également à celui de la parcelle. Cette section identifie les caractéristiques des parcelles que les acteurs certifient en priorité : modalités d'acquisition, usage qui en est fait, localisation.

\subsection{Mode d'acquisition de la parcelle}

La demande de certification est principalement induite par un besoin de sécurisation des transactions foncières : $62 \%$ des parcelles certifiées ont été achetées (alors que les achats représentent $37 \%$ des parcelles de l'échantillon ${ }^{11}$ ), $38 \%$ des parcelles achetées sont certifiées (tab. A en annexe). Dans l'optique des intéressés, sécuriser un achat revient à se prémunir contre une remise en cause de la transaction par le cédant lui-même, par ses héritiers après son décès ou encore par un tiers se revendiquant propriétaire de la parcelle. La plus faible certification des parcelles héritées est justifiée par les enquêtés par les relations de confiance intrafamiliale, limitant les risques de contestation des droits. De surcroit, si ces terres héritées font partie d'un patrimoine familial depuis plusieurs générations, l'héritier est a priori assuré d'être reconnu comme propriétaire légitime. Les membres de la famille ainsi que les autres villageois peuvent témoigner en sa faveur en cas de contestation d'un tiers. Dans certaines familles toutefois, la confiance et la «connaissance commune » relative aux droits des uns et des autres ne sont plus perçues comme une source suffisante de sécurisation : $22 \%$ des parcelles certifiées ont été héritées, $15 \%$ des héritages sont certifiés (tab. A). Certains enquêtés expliquent également avoir certifié une parcelle héritée pour se prémunir contre le risque qu'un autre héritier n'empiète sur la parcelle lorsque lui-même certifie sa part. Le cas d'étude rappelle ainsi - même s'il ne s'agit pas de la motivation dominante - comment la famille peut aussi être source d'insécurité foncière. De façon générale, les parents justifient la certification par le souci de préserver l'intérêt de leurs descendants, même s'il n'existe pas de crainte spécifique au moment présent. Ils cherchent à

11 Les transactions s'inscrivent dans $57 \%$ des cas dans le cadre familial, pour $15 \%$ dans une relation amicale ou de voisinage et, à $28 \%$, sont réalisées avec un individu non résidant du village et non apparenté. 
éviter les conflits tant entre les héritiers et des personnes extérieures à la famille, qu'entre héritiers.

À l'inverse, certains individus en âge de transmettre la terre - ou assez âgés pour anticiper un proche transfert - n'envisagent pas de certifier, considérant que la procédure de transmission sera plus lourde si le certificat est établi à leur nom. Ils préfèrent qu'à terme leurs héritiers soient ceux qui décident de la certification. Les enfants, quant à eux, attendent un partage officiel pour certifier en leur nom (cf. 3.2.). Les donations ne représentent que $3 \%$ des terres possédées, mais $6 \%$ des parcelles certifiées (tab. A). Ces parcelles sont certifiées afin de se prémunir des risques de contestation de membres de la fratrie, lorsque le cédant est un parent, ou des enfants du donateur, lorsque la donation intervient dans un cadre familial plus large.

Enfin, les terres acquises à la suite d'un travail de défrichement représentent $8 \%$ des terres en possession de l'échantillon et leur proportion n’apparaît pas plus élevée parmi le sous-échantillon de terres certifiées (tab. A). La présence de villageois témoins de cet investissement est en général jugée sécurisante par les enquêtés. La demande de certificat peut cependant être justifiée lorsque la terre défrichée est localisée sur les collines ; il s'agit alors de délimiter clairement la parcelle afin d'éviter les empiètements de la part des voisins. L'étape de la procédure de certification qui consiste à préciser sur le terrain les limites de la parcelle avec les agents du guichet foncier et des témoins est particulièrement rassurante pour les usagers. Le report, sur le certificat, du plan de la parcelle est considéré par certains enquêtés comme équivalent au bornage d'une parcelle qui aurait été titrée.

\subsection{Usage et localisation de la parcelle}

Quatre types de parcelles peuvent être distingués, selon l'usage qui en est fait : les rizières situées dans les bas-fonds, les tanety, les rizières irriguées situées sur les collines et les terrains situés en bas de colline sur lesquels sont généralement bâties les maisons. Plus de 70 \% des parcelles certifiées sont des rizières de bas-fonds, alors qu'elles représentent seulement $55 \%$ de l'échantillon (tab. A). Les propriétaires certifient principalement les parcelles qu'ils valorisent le plus, tout particulièrement au regard de leur importance en termes de sécurité alimentaire. Les tanety ont généralement une valeur 
(marchande et d'usage) nettement moindre que celle des rizières, sauf lorsqu'elles sont aménageables en rizière irriguée car situées à proximité d'une source d'eau.

La superficie moyenne des rizières ${ }^{12}$ est de 8,2 ares lorsqu'elles sont certifiées, de 6,6 ares dans le cas contraire (tab. A). Les enquêtés sécurisent en priorité les plus grandes rizières - qui contribuent davantage à l'autoconsommation - d'autant que la majorité des rizières a une superficie inférieure à 30 ares, seuil de la première tranche de tarification de la procédure de certification $^{13}$. La plus faible certification des terrains bâtis est justifiée par la sécurisation apportée par la présence de la maison. Les parcelles certifiées sont détenues depuis moins longtemps. Les personnes qui les exploitent depuis un grand nombre d'années ont en général l'assurance d'être reconnues comme propriétaires légitimes, les risques de contestation étant susceptibles d'être moins forts que dans le cas d'une parcelle nouvellement possédée. La localisation des parcelles influe également sur le sentiment d'insécurité foncière. Les parcelles les plus éloignées du domicile sont significativement plus certifiées (tab. A). Le risque d'empiètement ou de mise en culture par un tiers opportuniste est évoqué dans le cas de parcelles trop éloignées pour permettre une surveillance quotidienne. Il s'agira tout particulièrement de parcelles de tanety, qui sont alors parfois moins bien entretenues et peuvent être vues comme abandonnées. La crainte d'un empiètement des limites de la parcelle est plus forte si les relations avec les exploitants voisins ne sont pas bonnes. Dans ce dernier cas, certains enquêtés craignent également que leur parcelle puisse être certifiée par un voisin. Certains justifient leur demande de certificat par le fait qu'il existe d'ores et déjà des litiges entre les exploitants voisins et qu'ils craignent une extension de ces contestations sur leur parcelle.

12 Les superficies des tanetyétant difficilement évaluables par les enquêtés, nous ne les avons pas documentées; seules les superficies des rizières sont renseignées dans notre base de données.

13 Le coût d'un certificat s'élevaient, en 2011, à 3000 ariary de frais fixes, auxquels il fallait ajouter 28000 ariary pour les superficies inférieures à 30 ares, 38000 ariary pour les superficies comprises entre 30 et 50 ares, 48000 ariary pour celles comprises entre 50 et 90 ares, et 5 ariary $/ \mathrm{m}^{2}$ pour les parcelles d'une superficie supérieure à 90 ares. 
Les sources et types d'insécurité foncière (de perception d'insécurité foncière) apparaissent ainsi variés, et appréhendables tout particulièrement au niveau de la parcelle. Ce constat témoigne de la difficulté de mesurer la sécurité ou l'insécurité foncière pour des traitements formels de données (Arnot et al., 2011) et questionne la pratique usuelle consistant à établir des indicateurs de sécurité/insécurité foncière au seul niveau du ménage.

\section{Un faible recours à la certification : éléments d'interprétation}

Malgré les campagnes d'information et d'incitation menées par les agents du guichet foncier ${ }^{14}$, moins de $20 \%$ des ménages de la commune disposent d'au moins un certificat. Cette section propose une interprétation de ce constat, en envisageant diverses raisons possibles.

\subsection{La certification foncière : une procédure méconnue ou trop complexe ?}

Au moment des enquêtes, quatre années après la mise en place du guichet foncier, les populations avaient connaissance de l'existence du programme de certification (c'était en particulier le cas de $80 \%$ des ménages de notre échantillon n'ayant demandé aucun certificat foncier ${ }^{15}$ ). Bien que certains individus soient restés initialement prudents quant à leur décision d'entrer dans la procédure de certification, les craintes envers le nouveau service communal se sont apaisées au cours des toutes premières années de fonctionnement. Dans notre échantillon, 2 \% seulement des cas de parcelles non certifiées sont justifiés par un manque de confiance envers le guichet ou ses agents (tab. 2).

14 Une période de "promotion " a été organisée pendant un mois dans tous les fokontany en 2007, en 2009 et en 2011, avec une réduction de 50 \% du coût de la certification.

15 Un défaut d'information n'est susceptible d'expliquer que la situation de 25 des 139 enquêtés ne disposant pas de certificat. Sur ces 25 personnes, 15 sont des femmes; ces dernières étant généralement moins informées que les hommes. 
Tableau 2. Raisons avancées par les enquêtés justifiant l'absence de certificat foncier

\begin{tabular}{lc} 
Raison justifiant l'absence de certificat & Nombre de parcelles \\
\hline Manque d'argent & $482(52 \%)$ \\
\hline Absence de crainte de contestation & $152(17 \%)$ \\
\hline Manque de temps & $144(16 \%)$ \\
\hline Incomplétude du faisceau de droits & $85(9 \%)$ \\
\hline Parcelle hors de compétence du guichet & $38(4 \%)$ \\
\hline Manque de confiance envers le guichet & $16(2 \%)$ \\
\hline Total & $1193(100 \%)$ \\
\hline
\end{tabular}

Source : Données issues des questionnaires (Boué, 2013).

Il est souvent fait état, dans la littérature, de procédures de formalisation légale favorisant les individus les plus éduqués. Dans le cas de Faratsiho, le niveau d'éducation n'apparaît pas comme déterminant de la demande de certificat, des ménages ne maîtrisant ni la lecture ni l'écriture ayant eu accès à des certificats fonciers ${ }^{16}$ (Boué, 2013). La procédure de certification est beaucoup plus simple que celle de l'immatriculation et est bien perçue comme telle. Sur les 203 enquêtés disposant d'au moins un certificat, $61 \%$ la jugent pas ou peu compliquée, 25 \% moyennement compliquée et $14 \%$ compliquée ou très compliquée. La proximité du guichet (comparativement aux services des Domaines) est appréciée des enquêtés : pour les résidents les plus éloignés, l'aller-retour est possible en une journée à pied, alors que se rendre aux services des Domaines à Antsirabe demande au minimum deux jours et induit des dépenses. La décentralisation de la gestion foncière fournit un réel service de proximité.

\subsection{Des parcelles non certifiables : cadre réglementaire et règles locales}

La non certification de parcelles s'explique parfois (13\% des cas) par l'impossibilité de certifier du fait des contraintes du cadre réglementaire ou au regard des normes locales (tab. 2). Certaines parcelles ne peuvent pas être certifiées lorsqu'elles sont situées hors du domaine de compétence du guichet, étant d'ores et déjà titrées ou en cours de titrage - une situation qui concerne un nombre très limité de cas (38 parcelles sur 1193 non certifiées,

16 Aucune de nos variables relatives aux niveaux d'instruction n'apparaît significative dans les tests formels. 
soit $4 \%$ ). L'immatriculation foncière a été peu pratiquée dans la commune de Faratsiho et touche quasi exclusivement des terrains des villages les plus « urbanisés » de la commune. De plus, la majorité de ces titres est établie au nom de personnes décédées ou inconnues des exploitants actuels, ces derniers possédant une fraction de parcelles titrées il y a de nombreuses années. Que le titre ait été délivré au nom d'une autre personne, ou que la procédure soit en attente depuis des années (blocage aux services des Domaines), la parcelle concernée est exclue de toute nouvelle reconnaissance légale.

Outre cette contrainte réglementaire, la question de la demande de certificat ne peut être traitée que relativement aux individus en mesure de l'exprimer légitimement, dans le contexte local. Nous suggérons ici d'enrichir le concept de faisceau de droits en intégrant - comme « fibre » potentielle du faisceau - le droit à certifier (ou plus largement à formaliser un droit) comme action socialement autorisée. L'incomplétude du faisceau de droits du fait de l'absence de ce droit explique la non certification de 85 parcelles de notre échantillon (9\%) (tab. 2).

Le tableau 3 présente le faisceau de droits d'un individu au niveau de la parcelle, selon le mode d'accès à la possession ou à l'usage de cette dernière. Les enfants (généralement une fois mariés) peuvent bénéficier d'une délégation pérennisée de droits d'usage (non limitée à une campagne agricole). Les parents conservent alors des droits sur ces parcelles : ils peuvent opérer une redistribution des terres si un cadet se marie, si l'un des époux décède, si un choc survient dans la famille ou encore si un enfant revient au village. Ils peuvent également vendre la parcelle. La demande de certificat foncier au nom du bénéficiaire de droits délégués n'est pas envisagée. Les parents enquêtés craignent que les enfants puissent alors vendre la parcelle et conserver le fruit de la cession. Par ailleurs, un enfant obtenant un certificat en son nom est susceptible de remettre en cause les obligations associées à la délégation de droits (participation aux dépenses lors du famadihana ${ }^{17}$ ou pour le paiement de l'impôt). On a vu par ailleurs que des parents ne certifient pas certaines parcelles dont l'usage est délégué afin d'éviter d'alourdir la procédure de transfert aux descendants - particulièrement si la parcelle est

17 Le "retournement des morts " est une cérémonie familiale pratiquée dans les Hautes Terres lors de laquelle les ancêtres reçoivent un nouveau linceul. 
susceptible d'être partagée entre plusieurs héritiers. La demande de certificat est également exclue pour les parcelles héritées tant que le transfert n'a pas pris un caractère définitif. Certains enquêtés, après la mort de leurs parents, sont ainsi dans l'attente d'une réunion familiale pour avaliser la transmission de l'héritage, parfois difficile à organiser du fait de l'éloignement de certains membres de la famille.

Tableau 3. Le droit à certifier au sein du faisceau de droits

\begin{tabular}{|c|c|c|c|c|c|c|c|}
\hline \multirow{2}{*}{$\begin{array}{l}\text { Mode d'acquisition } \\
\text { des droits/ } \\
\text { types de droit }\end{array}$} & \multirow{2}{*}{ Achat ${ }^{18}$} & \multirow{2}{*}{ Défriche } & \multirow{2}{*}{$\begin{array}{l}\text { Donation } \\
\text { entre } \\
\text { vifs }\end{array}$} & \multicolumn{2}{|c|}{$\begin{array}{c}\text { Héritage des parents } \\
\text { décédés }\end{array}$} & \multirow{2}{*}{$\begin{array}{l}\text { Délégation } \\
\text { intra } \\
\text { familiale }\end{array}$} & \multirow[b]{2}{*}{ Prise en FVI } \\
\hline & & & & $\begin{array}{l}\text { Avant } \\
\text { partage } \\
\text { définitif }\end{array}$ & $\begin{array}{l}\text { Après } \\
\text { partage } \\
\text { définitif }\end{array}$ & & \\
\hline $\begin{array}{l}\text { Qualificatif général } \\
\text { des parcelles }\end{array}$ & \multicolumn{5}{|c|}{ Terres en possession } & \multicolumn{2}{|c|}{ Terres non possédées } \\
\hline Droit d'exploiter & Oui & Oui & Oui & Oui & Oui & Oui & Oui \\
\hline $\begin{array}{l}\text { Droit de déléguer le } \\
\text { droit d'exploitation } \\
\text { aux enfants }\end{array}$ & Oui & Oui & Oui & $\begin{array}{l}\text { Oui (avec } \\
\text { consensus } \\
\text { familial) }\end{array}$ & Oui & $\begin{array}{l}\text { Oui (avec } \\
\text { consensus } \\
\text { familial) }\end{array}$ & Non \\
\hline $\begin{array}{l}\text { Droit de céder } \\
\text { en location }\end{array}$ & Oui & Oui & Oui & Oui & Oui & \multirow{2}{*}{$\begin{array}{l}\text { selon les } \\
\text { « règles intra } \\
\text { familiales » }\end{array}$} & Non \\
\hline $\begin{array}{l}\text { Droit de céder } \\
\text { en métayage }\end{array}$ & Oui & Oui & Oui & Oui & Oui & & Non \\
\hline Droit de vendre & Oui & Oui & Oui & Non & Oui & Non & Non \\
\hline $\begin{array}{l}\text { Restrictions sur } \\
\text { le droit de vente }\end{array}$ & Non & Non & Non & - & Oui & - & - \\
\hline $\begin{array}{l}\text { Obligations envers } \\
\text { le cédant }\end{array}$ & Non & - & Possible & - & - & Oui & $\begin{array}{l}\text { Définies } \\
\text { selon } \\
\text { arrangement }\end{array}$ \\
\hline Droit de certifier & \multicolumn{3}{|c|}{ Oui } & Non & Oui & Non & Non \\
\hline
\end{tabular}

Source : Boué, 2013.

\subsection{La certification foncière : trop coûteuse ou non justifiée pour certaines parcelles?}

Le coût du certificat est de l'ordre de 25000 ariary, soit l'équivalent d'une vingtaine de kilogrammes de riz ou de dix journées de salariat agricole.

18 Les ventes sont complètes dans la zone d'étude, dans le sens où l'acquéreur n'a pas d'obligation envers le vendeur une fois la transaction réalisée. 
Ce coût varie selon la superficie des parcelles, mais la première tranche concerne les parcelles de moins de 30 ares et aucune des parcelles de l'échantillon n'atteint cette superficie. Une contrainte financière est évoquée pour $5^{2} \%$ des parcelles non certifiées lors des enquêtes par questionnaire (tab. 2). Le niveau de vie des ménages semble ici discriminant. Les enquêtés qui disposent d'au moins un certificat foncier sont mieux dotés en zébus et en matériel agricole (charrue, sarcleuse, etc.), exploitent une plus grande superficie de rizière, résident dans des maisons dont le toit est en tôle et non en chaume. Les couples sans aucun certificat pratiquent davantage le salariat agricole, ainsi que d'autres activités hors exploitation peu rémunératrices (tab. B en annexe). Bien que les plus aisés aient davantage accès à la procédure de certification, nos enquêtes montrent que les ménages susceptibles d'être les plus vulnérables ne sont pas exclus de la procédure. La contrainte financière joue moins sur le fait d'entrer dans le programme de certification que sur le nombre de parcelles concernées (tab. C en annexe). Les ménages les plus démunis effectuent un choix sous contrainte à effectuer et ils doivent établir des priorités quant aux parcelles à certifier.

Le coût de la certification, évoqué comme un facteur limitant dans les réponses des enquêtés par questionnaire ou, initialement, lors des entretiens apparaît vite, sur la base des entretiens plus approfondis comme masquant en fait l'absence d'intérêt d'une telle démarche pour une parcelle donnée. De fait, 83 des 143 enquêtés évoquant la contrainte de trésorerie avaient déjà certifié au moins l'une de leur parcelle. On notera également que les ménages sans certificat évoquent plus souvent un manque d’intérêt que les ménages en possédant au moins un, alors que la différence n’est pas significative relativement à la contrainte de trésorerie (tab. D en annexe). Certains considèrent en effet la certification comme une dépense injustifiée et certainement non prioritaire ${ }^{19}$, compte tenu des caractéristiques de la parcelle en question et de leur situation financière du moment.

19 La justification de l'absence de certificat sur une parcelle par le " manque de temps " - $16 \%$ des parcelles non certifiées (tab. A) - est également susceptible de traduire une absence d'incitation pour la certification du fait de l'absence de crainte de contestation. 


\subsection{Une sécurisation foncière par la certification ou par les dispositifs locaux?}

De nombreux enquêtés justifient l'absence de certification de certaines parcelles par l'absence de crainte de contestation de leurs droits, avec ici trois cas de figure : une relation de confiance existe avec le cédant, en cas d'achat ( $4 \%$ des 152 parcelles non certifiées du fait d'une absence de crainte) ; la possession de la parcelle est considérée comme relevant d'une « connaissance commune » indiscutable - particulièrement s'il s'agit d'une tanindrazana, littéralement la « terre des ancêtres » (46\% des parcelles) - ; l'enquêté dispose d'ores et déjà de documents (taratasy) sur la parcelle et les considère comme suffisants (50 \% des parcelles). Nous mettrons ici l'accent sur ce dernier argument. De nombreux travaux mentionnent le recours à des formes « informelles » ou « semi-formelles » d'écrit dans des contextes africains (Lavigne-Delville, 2002). À Madagascar, il s'agit d'actes sous-seing privé avec ou non des témoins, visés ou non par des autorités locales (du hameau), tamponnés ou non par le chef de fokontany et enregistrés ou non au niveau de l'arrondissement. Ces documents peuvent formaliser les transferts marchands (achat) ou non marchands (héritage, donation) de droits sur la terre ; ou attester de droits fondés sur la mise en valeur (Rabearimanana et al., 1994 ; PNF, 2007 ; Teyssier et al., 2007 ; Omrane, 2008). Tous les actes ne sont cependant pas équivalents et, dans la perception des acteurs, leur « force » varie selon l'instance qui valide l'acte. Le délégué d'arrondissement étant le représentant (accessible) le plus proche de l'autorité du fanjakana (la puissance publique), l'acte de vente qu'il délivre est pour cette raison perçu comme le document ayant le plus de « force »-comparativement à un acte tamponné par le chef de fokontany ou un acte sous-seing - en termes de sécurisation des droits (Boué et al., 2011). Cette procédure est connue de longue date et fait référence à un cadre de validation et d'arbitrage considéré comme relevant du fanjakana pour certains enquêtés. En définitive, de nombreuses parcelles ne sont pas certifiées lorsque le dispositif local d'ores et déjà mobilisé est considéré comme suffisant, compte tenu de la valeur de la parcelle $e^{20}$.

20 Sur la relation de complémentarité ou de substituabilité entre certification et procédures locales de sécurisation, voir Céline Boué et Jean-Philippe Colin (2015). 


\section{Conclusion}

Notre étude montre que la principale motivation pour entrer dans la procédure de certification est la recherche de sécurisation des droits sur la terre ; les résultats d'une enquête effectuée sur neuf autres communes malgaches (Burnod et al., 2014) convergent avec les nôtres. La perspective de mettre la terre sur le marché foncier dans de meilleures conditions ne joue pas ; les marchés fonciers sont actifs - et fortement développés dans le cadre familial -, mais les transferts de droits sont souvent sécurisés par d'autres « dispositifs » que le certificat (formalisation locale, relations interpersonnelles). La certification porte sur la sécurisation des achats (passés), mais ne semble pas participer au développement du marché foncier au moment des enquêtes. L'investissement est davantage contraint par le manque de capitaux que par l'absence d'une sécurisation légale et le certificat - dans le contexte étudié - ne facilite pas l'accès au crédit. Enfin, le fait que la principale motivation pour entrer dans la procédure de certification soit en définitive la recherche d'une meilleure sécurisation des droits sur la terre ne reflète pas une conflictualité foncière effective - en cela, notre étude conforte plusieurs travaux soulignant l'écart entre un faible nombre de litiges fonciers et la perception d'une insécurité par les ménages (Jacoby \& Minten, 2007 ; Jenn-Treyer \& Pouzoullic, 2008 ; Burnod et al., 2012). Les justifications de demande de certificat portent donc sur la réduction de risques envisagés : risques de remise en cause de transactions passées, risques induits par les transmissions intrafamiliales, risques de contestation de limites de parcelles ou encore crainte plus diffuse d'une possible contestation future des droits fonciers dans un contexte de saturation de l'espace. La certification intervient alors comme une assurance additionnelle, sur le long terme, de non contestation des droits sur une parcelle donnée, qui s’ajoute aux dispositifs locaux (Burnod et al., 2012).

Outre l'absence d'un contexte conflictuel ouvert au niveau de la commune, la faible demande de certification vient de ce que les ménages ne certifient pas l'ensemble des parcelles possédées. Notre investigation témoigne ainsi de l'intérêt d'une analyse des déterminants de la demande de certificat au niveau de la parcelle. Elle montre combien les stratégies de sécurisation dépendent des caractéristiques de cette dernière. En effet, les déterminants de la demande de certificat, outre les caractéristiques socio-économiques 
des ménages, reposent sur la combinaison de la valeur d'usage de la parcelle et de l'évaluation des risques de contestation dans le cadre des transferts de droits. Les acteurs certifient en priorité les parcelles auxquelles ils attachent le plus d'importance au regard de leurs conditions d'existence marquées par une forte précarité économique - en l'occurrence les rizières, qui contribuent à la sécurité alimentaire des ménages.

Le cas d'étude met également en évidence la nécessité d’une prise en compte des dispositifs locaux de sécurisation existants, dans l'appréciation des attentes à avoir d'un programme de reconnaissance légale des droits fonciers et dans les études d'impact de telles réformes foncières. L'analyse contextualisée de ces processus et dispositifs locaux de sécurisation devrait permettre une meilleure compréhension des caractères multiformes, subjectifs et surtout évolutifs de l'insécurité foncière, afin d'adapter le type d'offre de sécurisation légale.

\section{Céline Boué}

S'intéresse aux politiques foncières et aux stratégies de sécurisation des droits et des transferts fonciers à Madagascar et au Mexique. Ce travail de recherche sur la certification foncière à Madagascar a été réalisé pendant son doctorat à SupAgro, UMR Moisa, Montpellier.

Actuellement en post-doctorat à la Universidad Autonama Metropolitana CUAM - Xochimilco, Mexique), ses thèmes de recherche portent sur les maïs natifs et les chaînes de valeurs associées dans la vallée centrale de Mexico. celine.boue@gmail.com

\section{A récemment publié}

Boué C., Colin J.-P., 2015, "Formalisation légale des droits fonciers et pratiques de sécurisation des transactions dans les Hautes Terres malgaches ", Les Cahiers du Pôle foncier, n 10.

\section{Pierre-Marie Bosc}

Agro-économiste, chercheur au Cirad, UMR Moisa, travaille actuellement sur les transformations structurelles des agricultures, à l'origine de l'Initiative International World Agricultures Watch hébergée à la FAO. II a coordonné ou contribué à plusieurs ouvrages récents sur les transformations des agricultures familiales à travers le monde. II travaille également sur les dynamiques d'action collective. 


\section{A récemment publié}

Bosc P.-M., Sourisseau J.-M., Bonnal P. et al., (dir.), 2015, Diversité des agricultures familiales de par le monde. Exister, se transformer, devenir, Versailles, Éd. Quæ.

Bélières J.-F., Bonnal P., Bosc P.-M. et al. (dir.), 2015, Family Farming around the World. Defintions, Contributions and Public Policies, Paris/Montpellier, AFD/Cirad.

Bosc P.-M., Bélières J.-F., 2015, "Transformations agricoles : un point de vue renouvelé par une mise en perspective d'approches macro et microéconomiques ", Cahiers agricultures, vol. 24, n² 4, pp. 206-214.

\section{Jean-Philippe Colin}

Directeur de recherche à I'IRD, conduit depuis plusieurs décennies des investigations dans le champ du foncier rural (politiques foncières, droits de propriété, marchés fonciers, contrats agraires) dans une approche d'économie institutionnelle (Mexique, Côte d'Ivoire, Algérie).

\section{A récemment publié}

Tarrouth G., Colin J.-P., 2016, " Les acquisitions de terres rurales par les "cadres" en Côte d'Ivoire : premiers enseignements ", Cahiers agricultures, vol. 25, n 1, http://www.cahiersagricultures.fr/articles/cagri/abs/2016/01/ cagri160003-s/cagri160003-s.html (juin 2016).

Colin J.-P., 2014, "La concentration foncière par la tenure inversée (reverse tenancy) ", Études rurales, n 194, pp. 203-218, https://www.cairn.info/revueetudes-rurales-2014-2-page-203.htm (juin 2016).

Chauveau J.-P., Colin J.-P., 2014, "La question foncière à l'épreuve de la reconstruction en Côte d'Ivoire ", in Viti F. (dir.), La Côte d'Ivoire, d'une crise à l'autre, Paris, L'Harmattan, pp. 9-38. 


\section{BIBLIOGRAPHIE}

Alchian A. A., Demsetz H., 1973, "The Property Right Paradigm ", Journal of Economic History, vol. 33, $n^{\circ} 1$, pp. 16-27, http://www.jstor.org/stable/2117138 (juin 2016).

Arnot C. D., Luckert M. K., Boxall P. C., 2011, "What Is Tenure Security? Conceptual Implications for Empirical Analysis ", Land Economics, vol. 87, $\mathrm{n}^{\circ} 2$, pp. 297-311.

Atwood D., 1990, "Land Registration in Africa: The Impact on Agricultural Production ", World Development, vol. 18, n5, pp. 659-671, http://www. sciencedirect.com/science/article/ pii/0305750X90900160 (juin 2016).

Barrows R., Roth M., 1990, "Land Tenure and Investment in African Agriculture: Theory and Evidence ", Journal of Modern African Studies in Comparative International Development, vol. 28, n 2, pp. 265-297, http://www.jstor.org/ stable/160863 (juin 2016).

Besley T., 1995, "Property Rights and Investment Incentives: Theory and Evidence from Ghana ", Journal of Political Economy, vol. 103, n 5, pp. 903-937, http://www.jstor.org/stable/2138750 (juin 2016).

Boué C., 2013, Changement institutionnel et pratiques de sécurisation des droits fonciers. Le cas d'une commune rurale des Hautes Terres malgaches (Faratsiho), Thèse de doctorat, Montpellier, Montpellier SupAgro.

Boué C., Colin J.-P., Bignebat C., Bosc P.-M., 2011, " Déterminants de la certification foncière par les ménages malgaches: le cas d'une commune rurale des Hautes Terres Centrales ", 5 'Journées de recherches en sciences sociales, Dijon, Inra/Sfer/Cirad, 8-9 décembre.

Boué C., Colin J.-P., 2015, " Formalisation légale des droits fonciers et pratiques de sécurisation des transactions dans les Hautes Terres malgaches ", Cahiers du Pôle foncier, $\mathrm{n}^{\circ} 10$.

Burnod P., Andrianirina N., Boué C. et al., 2012, " Land Reform and Certification in Madagascar: Does Perception of Tenure Security Matter and Change ", Annual World Bank Conference, Washington, Banque mondiale, 24-27 avril.

Burnod P., Andrianirina N., Andrianirina Ratsialonana R. et al., 2014, La certification foncière au niveau ménages ruraux à Madagascar. Perception et effets (situation en 2011), Antananarivo, Observatoire du foncier, http://www.observatoire-foncier.mg/downloads/16-RapportPECF-version_Finale2.pdf (juin 2016).

Colin J.-P., 2008, " Disentangling IntraKinship Property Rights in Land: A Contribution of Economic Ethnography to Land Economics in Africa ", Journal of Institutional Economics, vol. 4, n² 2, pp. 231-254.

Colin J.-P., Ayouz M., 2006, "The Development of a Land Market? Insights from Côte d'Ivoire ", Land Economics, vol. 82, n³, pp. 404-423, http://www. jstor.org/stable/27647720 (juin 2016).

Colin J.-P., Le Meur P.-Y., Léonard E., 2009, "Introduction. Identifier les droits et dicter le droit : la politique des programmes de formalisation des droits fonciers", in 
Colin J.-P., Le Meur P.-Y., Léonard E. (dir.), Les politiques d'enregistrement des droits fonciers : du cadre légal aux pratiques locales, Paris, Karthala.

Deininger K., Feder G., 2009, " Land Registration, Governance, and Development: Evidence and Implications for Policy ", World Bank Research Observer, vol. 24, n², pp. 233-266, https://wbro. oxfordjournals.org/content/24/2/233. full? sid=4594d67b-9515-4063-be6602711b36f39c (juin 2016).

Droy I., Bidou J.-E., Rasolofo P., 2010, "Pauvreté et sécurisation foncière : les atouts et incertitudes d'une gestion décentralisée à Madagascar ", Taloha, $n^{\circ} 19$, http://www.taloha.info/document. php?id=867 (juin 2016).

Feder G., Onchan T., 1987, " Land Ownership Security and Farm Investment in Thailand ", American Journal of Agricultural Economics, vol. 69, $n^{\circ} 2$, pp. 311-320, http://www.jstor. org/stable/1242281 (juin 2016).

Feder G., Nishio A., 1999, "The Benefits of Land Registration and Titling: Economic and Social Perspectives ", Land Use Policy, vol. 15, n 1, pp. 25-43, http:// www.sciencedirect.com/science/article/ pii/S0264837797000392 (juin 2016).

Jacoby H., Minten B., 2007, "Is Land Titling in Sub-Saharan Africa Cost-Effective? Evidence from Madagascar ", The World Bank Economic Review, vol. 21, n³, pp. 461-485, http://wber. oxfordjournals.org/content/21/3/461. full? sid=4a7bba54-e6d4-4769-93d4$881027810 d c 8$ (juin 2016).

Jenn-Treyer O., Pouzoullic J., 2008, "Organisations paysannes et sécurisation foncière, quelle rationalité ? ", in Sandron F. (dir.), Population rurale et enjeux fonciers à Madagascar, Antananarivo/Paris, Cite/Karthala.

Lavigne-Delville P., 2002, "When Farmers Use "Pieces of Paper" to Record their Land Transactions in Francophone Rural Africa: Insights into the Dynamics of Institutional Innovation ", The European Journal of Development Research, vol. 14, n², pp. 89-108.

Migot-Adholla S. E., Hazelle P., Blarel B., et al., 1991, "Indigenous Land Rights Systems in Sub-Saharan Africa: A Constraint on Productivity? ", The World Bank Economic Review, vol. 5, $n^{\circ} 1$, pp. 155-175, (juin 2016).

Omrane M., 2008, Accès à la terre, dynamique démographique et ancestralité à Madagascar, Paris, L'Harmattan.

Ottino P., 1998, Les champs de l'ancestralité. Parenté, alliance et patrimoine, Paris, Karthala/Orstom.

Place F., Migot-Adholla S. E., 1998, " The Economic Effect of Land Registration on Smallholder Farms in Kenya: Evidence from Nyeri and Kakamega Districts ", Land Economics, vol. 74, n 3 , pp. 360-373, http://www.jstor.org/ stable/3147118 (juin 2016)

Platteau J.-P., 1996, "The Evolutionary Theory of Land Rights as Applied to SubSaharan Africa: A Critical Assessment ", Development and Change, vol. 27, $n^{\circ} 1$, pp. 29-86, http://onlinelibrary.wiley. com/doi/10.1111/j.1467-7660.1996. tb00578.x/epdf (juin 2016).

PNF, 2007, Guide pratique de la Gestion foncière décentralisée, Antananarivo, Ministère de l'Agriculture, de l'Élevage et de la Pêche). 
Rabearimanana G., Ramamonjisoa J., Ramiarantsoa H. R. et al., 1994, Paysanneries malgaches dans la crise, Paris, Karthala.

Schlager E., Ostrom E., 1992, " Property Rights Regimes and Natural Resources: A Conceptual Analysis ", Land EConomics, vol. 68, $n^{\circ} 3$, pp. 249-262, http://www.jstor.org/stable/3146375 (juin 2016).

Sjaastad E., Bromley D. W., 1997, « Indigenous Land Rights in Sub-Saharan Africa: Appropriation, Security and Investment Demand ", World Development, vol. 25, $\mathrm{n}^{\circ}$ 4, pp. 549-562, http://www.sciencedirect.com/ science/article/pii/S0305750X96001209 (juin 2016).

Smith R., 2004, "Land Tenure, Fixed Investment, and Farm Productivity: Evidence from Zambia's Southern Province ", World Development, vol. 32, $n^{\circ} 10, p p .1641-1661$, http://www. sciencedirect.com/science/article/pii/ S0305750X04001251 (juin 2016).
Teyssier A., Raharison H., Ravelomanantsoa Z., 2007, "La réforme foncière de Madagascar ou le pari de la compétence locale ", Réforme agraire, n 1, pp. 34-48.

Teyssier A., Ratsialonana R. A., Razafindralambo R. et al., 2009, « Décentralisation de la gestion foncière à Madagascar. Processus, innovations et premiers effets ", in Colin J.-P., Le Meur P.-Y., Léonard E. (dir), Les politiques d'enregistrement des droits fonciers. Du cadre légal aux pratiques locales, Paris, Karthala.

Van den Brink R., Thomas G., Binswanger H. et al., 2006, "Consensus, Confusion, and Controversy. Selected Land Reform Issues in Sub-Saharan Africa ", World Bank Working paper $n^{\circ} 71$, Washington, Banque mondiale, http:// hdl.handle.net/10986/7387 (juin 2016).

Zombre U., 2013, Les marchés du crédit et de la terre au lac Alaotra (Madagascar). Interactions et déterminants de la participation des ménages, Thèse de doctorat, Montpellier, Cirad/Université Montpellier 1. 


\section{ANNEXES}

\section{Tableau A. Caractéristiques des parcelles et certification ${ }^{21}$}

\begin{tabular}{|c|c|c|c|c|c|c|c|}
\hline \multirow[t]{2}{*}{$\begin{array}{c}\text { Caractéristiques des parcelles } \\
\text { certifiables }\end{array}$} & \multicolumn{2}{|c|}{$\begin{array}{l}\text { Parcelles } \\
\text { certifiées }\end{array}$} & \multicolumn{2}{|c|}{$\begin{array}{c}\text { Parcelles } \\
\text { non certifiées }\end{array}$} & \multicolumn{2}{|c|}{$\begin{array}{c}\text { Parcelles } \\
\text { en possession } \\
\text { dans la zone } \\
\text { de compétence } \\
\text { du guichet }\end{array}$} & \multirow[t]{2}{*}{ Test } \\
\hline & moy & $e-t$ & moy & $e-t$ & moy & $e-t$ & \\
\hline 1 si la parcelle a été mise en valeur & 0,09 & $\mathrm{O}, \mathrm{O} 1$ & o,o8 & $\mathrm{O}, \mathrm{O} 1$ & 0,08 & O,oO1 & Non \\
\hline 1 si la parcelle a été héritée & 0,22 & $\mathrm{O}, \mathrm{O} 2$ & 0,55 & 0,02 & 0,51 & 0,02 & $* * *$ \\
\hline $\begin{array}{l}1 \text { si la parcelle a été reçue } \\
\text { en donation }\end{array}$ & o,o6 & $\mathrm{O}, \mathrm{O} 2$ & $\mathrm{O}, \mathrm{O} 2$ & 0,001 & 0,03 & 0,001 & * \\
\hline 1 si la parcelle a été achetée & 0,62 & o,03 & 0,34 & $\mathrm{O}, \mathrm{O} 2$ & 0,37 & $\mathrm{O}, \mathrm{O} 2$ & $* * *$ \\
\hline $\begin{array}{l}1 \text { si la parcelle est une terre } \\
\text { de colline }\end{array}$ & 0,19 & $\mathrm{O}, \mathrm{O} 2$ & 0,31 & $\mathrm{O}, \mathrm{O} 2$ & 0,29 & O,O2 & $* * *$ \\
\hline $\begin{array}{l}1 \text { si la parcelle est une rizière } \\
\text { de bas-fonds }\end{array}$ & 0,76 & $\mathrm{O}, \mathrm{O} 2$ & $0,5^{2}$ & $\mathrm{O}, \mathrm{O} 2$ & 0,55 & $\mathrm{O}, \mathrm{O} 2$ & $* * *$ \\
\hline 1 si la terre est constructible & 0,08 & 0,01 & 0,19 & $\mathrm{O}, \mathrm{O} 2$ & 0,17 & $\mathrm{O}, \mathrm{O} 1$ & $* * *$ \\
\hline 1 si la terre de colline est irriguée & $\mathrm{O}, \mathrm{O} 3$ & o,oO1 & o,013 & o,oO1 & $\mathrm{O}, \mathrm{O} 2$ & o,oo1 & $* *$ \\
\hline 1 si la terre est une rizière héritée & 0,17 & O,02 & 0,25 & $\mathrm{O}, \mathrm{O} 2$ & 0,24 & $\mathrm{O}, \mathrm{O} 2$ & $* * *$ \\
\hline Durée détention (nombre années) & 19,6 & 0,74 & 22,4 & 0,53 & 22,1 & 0,48 & $* * *$ \\
\hline $\begin{array}{l}1 \text { si la terre est à moins de } 15 \mathrm{~min} \\
\text { de marche }\end{array}$ & 0,73 & $\mathrm{O}, \mathrm{O} 2$ & 0,8 & $\mathrm{O}, \mathrm{O} 1$ & 0,78 & $\mathrm{O}, \mathrm{O} 1$ & $* * *$ \\
\hline Nombre total de parcelles & \multicolumn{2}{|c|}{407} & \multicolumn{2}{|c|}{969} & \multicolumn{2}{|c|}{1376} & \\
\hline Superficie rizière (ares) & 8,2 & 0,18 & 6,6 & 0,14 & 7,6 & 0,11 & $*$ \\
\hline Nombre total de rizières & \multicolumn{2}{|c|}{369} & \multicolumn{2}{|c|}{627} & \multicolumn{2}{|c|}{996} & \\
\hline
\end{tabular}

${ }^{*} p<0.1,{ }^{* *} p<0.05,{ }^{* * *} p<0.01$

Source : Données issues des questionnaires (Boué, 2013 ; données pondérées).

21 Sur la base de variables dichotomiques (1 ou 0), on teste la significativité des différences en moyenne entre parcelles certifiées et non certifiées au moment de l'enquête. 
Tableau B. Caractéristiques des ménages et certification

\begin{tabular}{|c|c|c|c|c|c|c|c|}
\hline \multirow[t]{2}{*}{$\begin{array}{l}\text { Caractéristiques } \\
\text { des ménages }\end{array}$} & \multicolumn{2}{|c|}{$\begin{array}{c}\text { Ménages } \\
\text { avec au moins } \\
\text { un certificat } \\
\text { foncier }\end{array}$} & \multicolumn{2}{|c|}{$\begin{array}{c}\text { Ménages } \\
\text { sans certificat } \\
\text { foncier }\end{array}$} & \multicolumn{2}{|c|}{$\begin{array}{l}\text { Ménages de } \\
\text { l'échantillon } \\
\text { avec terres en } \\
\text { possession }\end{array}$} & \multirow[t]{2}{*}{ Test } \\
\hline & moy & $e-t$ & moy & e-t & moy & e-t & \\
\hline $\begin{array}{l}\text { Possède au moins un vélo } \\
\text { ou un téléphone ou une radio }\end{array}$ & 0,57 & o,03 & 0,35 & 0,04 & 0,41 & o,03 & *** \\
\hline Maison dont le toit est en chaume & 0,63 & o,03 & 0,83 & o,o3 & 0,78 & O,02 & $* * *$ \\
\hline Maison dont le sol est en terre & 0,45 & o,03 & 0,58 & O,04 & 0,56 & 0,03 & $* * *$ \\
\hline Possède au moins une sarcleuse & 0,65 & 0,03 & 0,47 & 0,04 & $0,5^{2}$ & $\mathrm{O}, \mathrm{O} 3$ & $* * *$ \\
\hline Possède au moins une charrette & 0,46 & o,03 & 0,37 & O,04 & 0,39 & o,03 & * \\
\hline Possède au moins une charrue & 0,45 & o,03 & 0,34 & O,04 & 0,37 & o,03 & * \\
\hline Possède au moins un zébu & 0,71 & o,03 & o,06 & O,04 & 0,62 & o,03 & $* *$ \\
\hline $\begin{array}{l}\text { Pratique régulièrement le salariat } \\
\text { agricole }\end{array}$ & 0,34 & o,03 & 0,59 & O,04 & 0,53 & o,03 & *** \\
\hline $\begin{array}{l}\text { Pratique une activité peu } \\
\text { rémunératrice }\end{array}$ & 0,37 & o,o3 & 0,57 & 0,04 & $0,5^{2}$ & o,o3 & $* * *$ \\
\hline $\begin{array}{l}\text { Pratique une activité fortement } \\
\text { rémunératrice }\end{array}$ & 0,13 & O,02 & o,o8 & $\mathrm{O}, \mathrm{O} 2$ & 0,09 & $\mathrm{O}, \mathrm{O} 2$ & Non \\
\hline $\begin{array}{l}\text { Membre d'une institution } \\
\text { financière }\end{array}$ & 0,09 & $\mathrm{O}, \mathrm{O} 2$ & $\mathrm{O}, \mathrm{O} 2$ & $\mathrm{O}, \mathrm{O} 1$ & o,04 & $\mathrm{O}, \mathrm{O} 1$ & $* * *$ \\
\hline Superficie rizicole possédée (ares) & 27,8 & 1,9 & 16 & 1,2 & 19,1 & $1, \mathrm{O} 1$ & $* * *$ \\
\hline Nombre de rizières possédées & 4 & 0,15 & 3,1 & 0,14 & 3,3 & 0,11 & $* * *$ \\
\hline Nombre de ménages & \multicolumn{2}{|c|}{203} & \multicolumn{2}{|c|}{139} & \multicolumn{2}{|c|}{342} & \\
\hline
\end{tabular}

${ }^{*} p<0.1,{ }^{* *} p<0.05,{ }^{* * *} p<0.01$

Source : Données issues des questionnaires (Boué, 2013 ; données pondérées). 
Tableau C. Caractéristiques des ménages et nombre de certificats

\begin{tabular}{|c|c|c|c|c|c|c|c|}
\hline \multirow[t]{2}{*}{$\begin{array}{l}\text { Caractéristiques } \\
\text { des ménages }\end{array}$} & \multicolumn{2}{|c|}{$\begin{array}{l}\text { Ménages avec } \\
\text { au moins } \\
2 \text { certificats } \\
\text { foncier }\end{array}$} & \multicolumn{2}{|c|}{$\begin{array}{c}\text { Ménages } \\
\text { avec un seul } \\
\text { certificat } \\
\text { foncier }\end{array}$} & \multicolumn{2}{|c|}{$\begin{array}{c}\text { Ménages de } \\
\text { l'échantillon } \\
\text { avec au moins } \\
\text { 1 certificat }\end{array}$} & \multirow[t]{2}{*}{ Test } \\
\hline & moy & e-t & moy & e-t & moy & e-t & \\
\hline $\begin{array}{l}\text { Possède au moins un vélo } \\
\text { ou un téléphone ou une radio }\end{array}$ & 0,58 & $\mathrm{O}, \mathrm{O} 4$ & 0,57 & O,05 & 0,57 & $\mathrm{O}, 04$ & Non \\
\hline Maison dont le toit est en chaume & 0,61 & $\mathrm{O}, 04$ & 0,65 & o,05 & 0,63 & $\mathrm{O}, \mathrm{O} 3$ & Non \\
\hline Maison dont le sol est en terre & 0,4 & $\mathrm{O}, 04$ & 0,53 & O,04 & 0,45 & $\mathrm{O}, \mathrm{O} 3$ & * \\
\hline Possède au moins une sarcleuse & 0,73 & $\mathrm{O}, 04$ & 0,55 & 0,05 & 0,65 & $\mathrm{O}, \mathrm{O} 3$ & *** \\
\hline Possède au moins une charrette & 0,51 & 0,05 & 0,39 & o,05 & 0,46 & $\mathrm{O}, \mathrm{O} 3$ & * \\
\hline Possède au moins une charrue & 0,5 & o,05 & 0,38 & 0,05 & 0,45 & $\mathrm{O}, \mathrm{O} 3$ & $*$ \\
\hline $\begin{array}{l}\text { Le ménage possède au moins } \\
\text { un zébu }\end{array}$ & 0,73 & $\mathrm{O}, \mathrm{O} 4$ & 0,68 & 0,05 & 0,71 & $\mathrm{o}, \mathrm{O} 3$ & Non \\
\hline $\begin{array}{l}\text { Pratique régulièrement le salariat } \\
\text { agricole }\end{array}$ & 0,3 & o,04 & 0,4 & 0,05 & 0,34 & $\mathrm{o}, \mathrm{O} 3$ & * \\
\hline $\begin{array}{l}\text { Pratique une activité peu } \\
\text { rémunératrice }\end{array}$ & 0,38 & $\mathrm{O}, \mathrm{O} 4$ & 0,37 & o,05 & 0,37 & $\mathrm{o}, \mathrm{O} 3$ & Non \\
\hline $\begin{array}{l}\text { Pratique une activité fortement } \\
\text { rémunératrice }\end{array}$ & 0,17 & o,03 & 0,07 & o,o3 & 0,13 & $\mathrm{O}, \mathrm{O} 2$ & ** \\
\hline $\begin{array}{l}\text { Membre d'une institution } \\
\text { financière }\end{array}$ & o,o8 & $\mathrm{O}, 03$ & 0,1 & $\mathrm{o}, \mathrm{O} 3$ & 0,09 & $\mathrm{O}, \mathrm{O} 2$ & Non \\
\hline Superficie rizicole possédée (ares) & 32,2 & 2,6 & 22,1 & 2,8 & 27,8 & 1,9 & $* * *$ \\
\hline Nombre de rizières possédées & 4,6 & 0,2 & 3,2 & 0,2 & 4 & 0,15 & *** \\
\hline Nombre de ménages & & & & & & & \\
\hline
\end{tabular}

Source : Données issues des questionnaires (Boué, 2013 ; données pondérées). 


\section{Tableau D. Raisons justifiant l'absence de certificat exprimées par parcelle}

\begin{tabular}{|c|c|c|c|c|c|c|c|}
\hline \multirow[t]{2}{*}{$\begin{array}{c}\text { Raisons justifiant l'absence de } \\
\text { demande de certificat } \\
\text { sur au moins une parcelle } \\
\text { en possession }\end{array}$} & \multicolumn{2}{|c|}{$\begin{array}{l}\text { Ménages avec } \\
\text { au moins } \\
\text { un certificat } \\
\text { foncier }\end{array}$} & \multicolumn{2}{|c|}{$\begin{array}{l}\text { Ménages sans } \\
\text { certificat } \\
\text { foncier }\end{array}$} & \multicolumn{2}{|c|}{$\begin{array}{c}\text { Ménages } \\
\text { connaissant } \\
\text { le guichet } \\
\text { et ayant au } \\
\text { moins une } \\
\text { terre en } \\
\text { possession }\end{array}$} & \multirow[t]{2}{*}{ Test } \\
\hline & moy & e-t & moy & e-t & moy & e-t & \\
\hline Contrainte de trésorerie & 0,54 & $\mathrm{o}, \mathrm{O} 3$ & 0,6 & $\mathrm{o}, \mathrm{O} 5$ & 0,58 & $\mathrm{o}, \mathrm{O} 3$ & Non \\
\hline Manque de temps & 0,15 & $\mathrm{o}, \mathrm{O} 2$ & 0,13 & o,o3 & 0,14 & $\mathrm{O}, \mathrm{O} 2$ & Non \\
\hline $\begin{array}{l}\text { Absence de crainte } \\
\text { de contestation }\end{array}$ & o,o8 & $\mathrm{o}, \mathrm{O} 2$ & 0,17 & 0,04 & 0,15 & $\mathrm{o}, \mathrm{O} 3$ & $* *$ \\
\hline $\begin{array}{l}\text { Incomplétude du faisceau } \\
\text { de droits }\end{array}$ & 0,15 & $\mathrm{o}, \mathrm{O} 2$ & 0,17 & $\mathrm{o}, \mathrm{O} 3$ & 0,17 & $\mathrm{o}, \mathrm{O} 3$ & Non \\
\hline $\begin{array}{l}\text { Manque de confiance envers } \\
\text { le guichet }\end{array}$ & o & o & $\mathrm{O}, \mathrm{O} 3$ & $\mathrm{o}, \mathrm{O} 1$ & $\mathrm{o}, \mathrm{O} 2$ & $\mathrm{o}, \mathrm{O} 1$ & $* * *$ \\
\hline $\begin{array}{l}\text { Parcelle hors de compétence } \\
\text { du guichet }\end{array}$ & 0,07 & $\mathrm{o}, \mathrm{O} 2$ & o,08 & $\mathrm{o}, \mathrm{O} 2$ & o,08 & $\mathrm{o}, \mathrm{O} 2$ & Non \\
\hline
\end{tabular}

Source : Données issues des questionnaires (Boué, 2013 ; données pondérées). 